\title{
Guest Editorial: Multimedia for Cultural Heritage
}

\author{
Costantino $\operatorname{Grana}^{1} \cdot$ Giuseppe Serra $^{1}$
}

Published online: 12 March 2016

(C) Springer Science+Business Media New York 2016

Multimedia technologies have achieved impressive results in the last years and they may be the key for a revolution in the cultural heritage area. These new technologies in fact can now make available for the public huge amounts of heterogeneous data (e.g. Europeana) creating unbelievable opportunities of study and valorisation of the cultural items.

Moreover, the cultural sector is becoming a key tile in many national economic strategies. Recent reports of the Office of Travel and Tourism Industries in USA claim that more than $50 \%$ of the 40 million Americans traveling abroad visit historical places such as cultural heritage sites, art galleries and museums. The same interest is also found in Europe, South Asia and North Africa. Consequently, to deal with an increasing percentage of interest, there is a big effort from scientific communities and industries to propose new multimedia technologies to enjoy and enhance the cultural experience.

This special issue received 32 submissions demonstrating the great interest of this timely topic from the scientific community. After two rounds of revision, 15 manuscripts, which address very challenging issues of this field, have been accepted.

Saleh et al. (10.1007/s11042-014-2193-x) investigate the question "Who influenced this artist?" through the study of painting and artist similarity. In particular, they compare several distance measures between paintings, including Euclidean distance and a manifold-based distance, on a dataset with 66 artists and wide range of paintings.

The paper by Makantasis et al. (10.1007/s11042-014-2191-z) aims at describing an image filtering approach for large data collections, which is able to discard image outliers by using a combination of local visual descriptors, textual features, geo-tagging information and clustering techniques. The filtering approach is then used and tested on 3D reconstructions of archaeological sites.

Cappelletto et al. (10.1007/s11042-014-2065-4) propose a 3D reconstruction pipeline for cultural heritage objects suited to use consumer depth cameras as hand-held scanners. In particular, they exploit the color information acquired by the device not only for texturing, but also to improve the extraction of salient points and the geometry reconstruction. The paper by Ahn et al. (10.1007/s11042-015-2473-0) present an interactive scan planning approach specially

Costantino Grana

costantino.grana@unimore.it

Giuseppe Serra

giuseppe.serra@unimore.it

1 Dipartimento di Ingegneria "Enzo Ferrari”, University of Modena and Reggio Emilia, Modena, Italy 
designed for cultural heritage domain which helps the user to intuitively decide the next best position so as to build the geometry of historic monuments more quickly and more precisely.

Lucena et al. (10.1007/s11042-014-2063-6) present a classification technique that supports archaeologists in the categorization of wheel-made pottery pieces based on their profiles. In particular, each profile is defined as the cross section of the vessel in the direction of the rotational axis of symmetry and it is described by means of several Mathematical Morphology curves. Sfikas et al. (10.1007/s11042-014-2069-0) propose an approach for retrieval of complete 3D objects using range image queries that represent partial views.

Cohen et al. (10.1007/s11042-014-2190-0) present a system that assists the user in the process of reconstructing vessels by aligning 3D scanned fragments. Starting by a series of generic models constructed by the archaeologists, the approach is able to produce a virtual reconstruction of what the original vessel may have looked like, exploiting surface markings and colors information.

Three contributions of this special issue address the problem of film restoration. Stanco et al. (10.1007/s11042-014-2068-1) propose a method to detect and correct mistracking artifacts (a group of random rows of noise in the frame, usually white or black) in digitalized Analog videos. Rizzi et al. (10.1007/s11042-014-2064-5) propose a method for digital color, contrast and dynamic restoration to recover the appearance of color rather than the original color signal. In particular, this technique is based on a family of image enhancement algorithms called SCAs (for Spatial Color Algorithms) inspired by the capabilities of the Human Visual System.

Cultural Heritage archives are not only composed by visual items, such as images and videos, but they also contain audio and music resources. The paper by Silovsky et al. (10.1007/ s11042-014-2067-2) present their ongoing research focused on speaker recognition in historical oral archives. Based on a Czech Radio recoding data collected over the time span of more than 30 years, they presented the results of their investigation showing how several factors can affect the recognition process: the aging effect, variability of utterance durations, noise level, and the epoch of original broadcasting.

Lastly, several multimedia case studies and tools conclude the special issue. Karaman et al. (10.1007/s11042-014-2192-y) present the MNEMOSYNE system that builds a profile of the artworks of interest for each museum visitor based on passive observation. The resulting profiles are then used to provide personalized content delivery through a natural interaction interface on an interactive table. The paper by Bartolini et al. (10.1007/s11042-014-2062-7) proposes a recommender framework, able to deal with heterogeneous cultural heritage data, that provides to tourists personalized visiting paths. Real case studies in outdoor and indoor environments, the Paestum Ruins and Capodimonte Museum, are presented. Places et al. (10.1007/s11042-0142155-3) propose a framework for managing the workflow of the digitization of large collections of antique documents. In particular, they describe the digitization process, and a tool supporting all of its phases and tasks. The proposed solution is then applied and tested to the digitalization of more than 10,000 historical documents. Grana et al. (10.1007/s11042-014-2360-0) propose a system to segment text and illustration of digitized old documents exploiting a texture characteristic aimed at detecting the repeating patterns of text regions and differentiate them from pictorial elements. Finally, Lombardo et al. (10.1007/s11042-014-2066-3) present a multimedia tool suite for the annotation and visualization of the metadata of the dramatic heritage.

Acknowledgments We would like to thank the authors for submitting excellent contributions and for the great work done in the revision of their manuscripts. We are also immensely grateful to all the reviewers who have provided timely and constructive comments, which strongly improved many papers. Finally, our thanks go to the Editor-in-Chief, Prof. Borko Furht, and the Editorial Board of the Journal of Multimedia Tools and Applications for the exceptional effort they did throughout this process. 


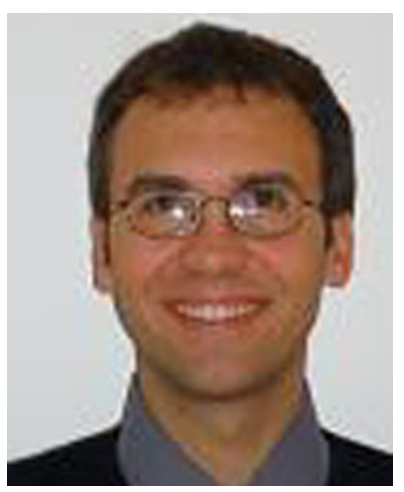

Costantino Grana graduated at the University of Modena, Italy in 2000 and achieved the Ph.D. in Computer Science and Engineering in 2004. He is currently Associate Professor at the Department of Engineering "Enzo Ferrari" of the University of Modena, Italy. His research interests are mainly in Computer Vision and Multimedia and include analysis and search of digital images of historical manuscripts and other cultural heritage resources, multimedia image and video retrieval, medical imaging, color based applications, motion analysis for tracking and surveillance. He co-organized the 14th International Conference on Image Analysis and Processing (ICIAP), two editions of the International Workshop Interactive Multimodal Pattern Recognition in Embedded SystemS (IMPRESS 2010, 2011), and two editions of the International Workshop on Multimedia for Cultural Heritage (MM4CH 2011, 2013). He published 5 book chapters, 25 papers on international peer-reviewed journals and 75 papers on international conferences. He is currently member of the IEEE, ACM and IAPR.

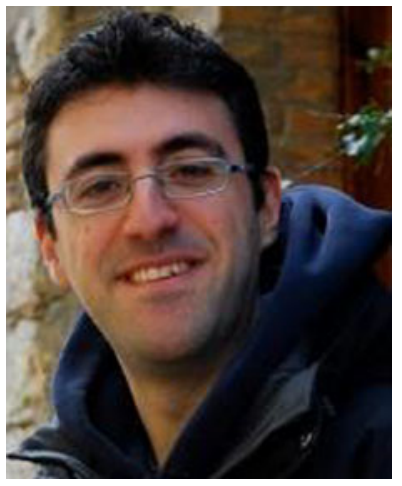

Giuseppe Serra graduated in Computer Engineering with honors from the University of Florence in 2006. He was awarded the title of Doctor of Philosophy in Computer Engineering, Multimedia and Telecommunications in 2010 from The MICC Center (Center of Excellence in the area of new media established by the MIUR). Currently he is Assistant Professor at University of Modena and Reggio Emilia (Italy). He was a visiting scholar at Carnegie Mellon University, Pittsburgh, PA, and at Telecom ParisTech/ENST, Paris, in 2006 and 2010 respectively. His research interests include image and video analysis, multimedia ontologies and multiple view geometry. He has published more than 40 publications in scientific journals and international conferences. He has been awarded the best paper award by the ACM\&SIGMM Workshop on Social Media in 2010. 